\title{
Integrated care facilitation model reduces use of hospital resources by patients with pediatric asthma
}

Bird, Stephen; Noronha, Michelle; Kurowski, William; Orkin, Carl; Sinnott, H

https://researchrepository.rmit.edu.au/esploro/outputs/9921858268901341/filesAndLinks?institution=61RMIT_INST\&index=null

Bird, S., Noronha, M., Kurowski, W., Orkin, C., \& Sinnott, H. (2012). Integrated care facilitation model reduces use of hospital resources by patients with pediatric asthma. Journal for Healthcare Quality, 34(3), 25-33. https://doi.org/10.1111/j.1945-1474.2011.00143.x

Document Version: Accepted Manuscript

Published Version: https://doi.org/10.1111/j.1945-1474.2011.00143.x

Repository homepage: https://researchrepository.rmit.edu.au

(C) 2011 National Association for Healthcare Quality

Downloaded On 2023/04/26 19:21:45 +1000 
Thank you for downloading this document from the RMIT Research Repository.

The RMIT Research Repository is an open access database showcasing the research outputs of RMIT University researchers.

RMIT Research Repository: http://researchbank.rmit.edu.au/

\section{Citation:}

Bird, S, Noronha, M, Kurowski, W, Orkin, C and Sinnott, H 2012, 'Integrated care facilitation model reduces use of hospital resources by patients with pediatric asthma', Journal for Healthcare Quality, vol. 34, no. 3, pp. 25-33.

See this record in the RMIT Research Repository at:

https://researchbank.rmit.edu.au/view/rmit:16014

Version: Accepted Manuscript

Copyright Statement: (c) 2011 National Association for Healthcare Quality

Link to Published Version:

http://dx.doi.org/10.1111/j.1945-1474.2011.00143.x

PLEASE DO NOT REMOVE THIS PAGE 
Integrated care facilitation model reduces use of hospital resources by patients with paediatric asthma 


\begin{abstract}
This evaluation assessed a model of care for Paediatric Asthma patients that aimed to promote health and reduce their preventable and/or avoidable use of acute hospital services. Paediatric asthma patients $(n=223)$ were allocated Care Facilitators who provided assistance in the promotion of carer/self-management, education and linkage to an integrated the healthcare system, comprising of acute and community based healthcare providers. Patients' use of acute hospital services (Emergency Department presentations, Admissions and Bed-days) pre- and post-recruitment were compared using Wilcoxon signed rank tests. The Paediatric Asthma Care Givers Quality of Life Questionnaire' was used to assess changes in health and quality of life. The patients displayed a $57 \%$ reduction in Emergency Department Presentations, 74\% in Admissions and a 71\% reduction in bed-days. Whereas a comparator group displayed $27 \%, 32 \%$ and $14 \%$ increases respectively. Patients also reported significant improvements in quality of life domains of: Activity Limitation $(+5.6, \mathrm{p}<0.001)$ and Emotional function $(+9.1, \mathrm{p}<0.001)$. The reduction in the use of Hospital Services was attributed to the aversion of preventable presentations and admissions, via the enhancement of carer/self-management and access to community health services. These outcomes were supported by indicators of improved patient health and quality of life, and comments by the participant's carers.
\end{abstract}

Key words: Paediatric Asthma, Care facilitation, Model of care, Emergency department presentations 


\section{Background}

In recognition of the growing demand on its hospital services, between 2001 and 2005 the Victorian State Government (Australia) invested \$582 million into the implementation of a 'Hospital Demand Management' strategy. Of this, \$150 million were for projects aimed at reducing the demand on hospital services and improving patient health through new approaches to patient management (Department of Human Services, 2006). These projects were collectively known as the Hospital Admissions Risk Programme (HARP) and in the 4 years immediately following its establishment, further funding (\$53 million) was provided in 2005-06 to enable the successful models of care to be implemented and delivered more broadly across the state (Department of Human Services, 2006). This paper describes the patient and hospital demand outcomes of a project that was established by a consortium (Table 1) of acute and community health care providers, based in the western suburbs of Melbourne, for patients with Paediatric Asthma and their carers.

\section{Project description and model of care}

Paediatric Asthma patients were identified as a group who frequently presented to hospital Emergency Departments (ED), and for whom it was perceived that their use of hospital services could be reduced and general health improved via the implementation of a new model of care. The rationale for targeting this group was a perception that some of their presentations and subsequent hospital admissions could be prevented by the patients' carers, and for the older children the patients themselves, improving their 
understanding of their condition, better carer or self-management of their condition (Clark et al., 2009, Spiegel et al., 2006), effective use of care and action plans, and facilitated access to community based health services (Department of Human Services 2003; Department of Health and Ageing 2005). These perceptions concurred with the findings of Reeves et al. (2006) who in a US study found that it was common for the carers of asthmatic children to not possess a spacer or written action plan, not to attend follow-up appointments after an Emergency Department (ED) visit, and to go directly to ED when needing asthma care.

Other factors liable to be contributing to this overuse of the acute sector services in the region include its socio-economic demographic, with areas characterised with mild socioeconomic disadvantage (Department of Human Services, 2005; Australian Bureau of Statistics, 2001). Consequently, many of the presenting patients were from low income families who viewed the hospital as a free or low cost service. This phenomenon of overusing the hospital emergency departments was likely to have been accentuated by the relatively low number of General Practitioners and other health providers in the region (Australian Institute of Health and Welfare, 2003) and a decreasing number of General Practitioners in the region who 'bulk bill', since it has been reported that low-income clients, who did not wish to pay for services, are disinclined to visit their GP and are more likely to present to the hospital, even if they would have been reimbursed eventually (Hopkins and Speed, 2005). (The policy of bulk billing provides free GP services for the patient at the point of delivery and time of service, the alternative is to charge the patient who then seeks a rebate). Furthermore, the region is the most culturally 
diverse area in Victoria (Department of Human Services, 2002; Australian Bureau of Statistics, 2001) with over one third of the region's population having been born overseas, or speaking a language other than English at home. Consequently many people from the area experience difficulties in understanding the health care system, the options available to them, and how to access these services. Inevitably therefore, the local acute hospitals, being very visible institutions, are seen by them as the primary locations for seeking all health advice and intervention.

Hence a key objective of the model of care was to breakdown the 'silo' structure of the existing healthcare system, whereby each organisation had separate funding and commonly operated with only limited linkages to other health organisations within the region. In particular, effective referral between acute and community healthy providers was deemed essential for this patient group as it would generate coordinated access to all services, and should produce a 'joined-up' and coherent health service. This was achieved through project specific funding and the involvement of senior staff from each organisation on the 'Governance Committee'. This thereby ensured that decisions concerning funding and resources could be made by the committee, and would then be implemented by their respective organisations. The importance of such "Leadership Support" and dedicated funding have been highlighted by Parrish et al. (2009) for the sustainability of such interventions. Additionally, the employment of staff (Care Facilitators) at both acute and community based health organisations, actively ensured linkage between health service organisations, and the Care Facilitators were able to link 
patients directly to these services as well as ensuring that their carers were aware of how to continue accessing them as required in the future.

Hence many of the elements of the model concurred with the requirements for effective 'Care Transition' (“...the movement of patients from one healthcare practitioner or setting to another...”, Parrish et al., 2009), which has been identified as an important factor with the potential to reduce preventable events and readmissions. In particular previous work has highlighted the risks of poor Care Transition through discontinuity between the different components of the health-care system (Kripalani et al. 2007) and has emphasised the importance of collaborative hospital-community partnerships (Parrish et al. 2009). Other identified key elements for effective Care Transition include: communication (Kripalani et al. 2007), consideration of the needs of diverse communities (Parrish et al. 2009), engagement with social support systems (Kripalani et al. 2007), and a means to address patient difficulties with complex discharge instructions, medication management and self-care responsibilities (Kripalani et al. 2007), all of which received attention in the model of care evaluated here.

\section{Recruitment to the model of care}

Recruitment was through the identification of frequent presenters (two or more presentations in the previous 12 months) to the hospitals' ED departments, or in a few cases patients identified as being at risk of future frequent presentations. A flagging system in the hospital records was used for patients fulfilling these criteria, and Care Facilitators were notified upon their next presentation to ED. A Care Facilitator then 
contacted the patient's carer in the hospital, or if already discharged, by telephone. The Care Facilitator invited them to participate in the new model of care, and if they/their carer agreed to participate, written informed consent was provided.

\section{Model of Care}

The project team for the model of care included multi-skilled Care Facilitators with professional expertise in nursing and asthma education. The details of related projects have been described elsewhere (Smith et al., 2003), but in summary comprised of:

1. A 'Gateway System'. Recruitment (as described above)

2. Disease specific streams. Patients were allocated to the Paediatric Asthma project stream, which was managed by specialist medical practitioners and nurses.

Decisions concerning strategies for the stream were discussed and reported at the stream steering committee, which comprised of the medical practitioners, GPs, school nurses, care facilitators, community health staff, project managers and evaluators.

3. Assessment of needs. A Care Facilitator performed a comprehensive assessment either in the hospital or in the patient's home. This included the Paediatric Asthma Care Givers Quality of Life Questionnaire' (PACQLQ) and/or the Paediatric Asthma Quality of Life Questionnaire' (PAQLQ) for the older children (Juniper et al., 1996a, 1996b).

4. Care coordination and facilitation. The results of each patient's assessment were used to identify issues for the patient, barriers to the effective management of their health, and factors putting the participant at risk of preventable events and 
avoidable presentations to the hospital. The assessment results were taken to a multidisciplinary case conference and this information was then used as a basis for the individual care plan.

5. Education and action plans. Care Facilitators provided asthma education for carers and patients, including the correct use of preventer medicines, spacers, and the provision of written asthma action plans. They also arranged follow up sessions with the carer and patient, which through being proactive were intended to overcome some of the barriers associated with a low Primary Care follow-up (Zorc et al., 2005), and the risk of preventable events in this patient group.

6. Facilitated access to a suite of services. The Care Facilitators then facilitated the patient's and carer's access to the health services they required. Examples of services arranged included primary health care assessments, psychology services, occasional child care for siblings whilst follow up appointments were arranged, housing assistance and financial assistance for medical equipment.

In the process of achieving the desired level of facilitation and self/carer-management, the Care Facilitators would arrange between 4 and 7 appointments with the patient/carer, with most of these occurring in the patients' home, although some could take place in the GP surgery or outpatient clinic if considered more appropriate. Patients were discharged from the project when it was deemed that optimal self/carer-management had been achieved

\section{Methods of evaluation}


Assessment of usage of acute hospital services

The patients' rates of Emergency Department (ED) presentations, Inpatient Admissions and Inpatient Bed-Days before and after their recruitment were calculated from the Hospitals' patient activity records. A 'Comparator Group' was generated from patients who had presented with the same characteristics in the 3 years prior to the inception of the new model of care (comparator group; $n=72$ ). For the Comparator Group a 'dummy recruitment' date was allocated to each patient using the date of separation from their second presentation in 12-months, which under the new model of care would be the event to have initiated contact by a Care Facilitator. The Comparator Group was composed of 45 male and 27 female patients, aged $5.1 \pm 4.3$ years (range 1 to 18 years). They had an average of $3.6 \pm 2.0 \mathrm{ED}$ presentations within the period of 12 months prior to their dummy recruitment and by comparison, the HARP-Asthma group ( $\mathrm{n}=223$ ), had an average of $3.0 \pm 1.7$ ED Presentations prior to recruitment. Kruskal-Wallis tests did not find any significant differences between the HARP intervention and Comparator groups with regard to standardised PRE-recruitment/dummy recruitment rates of ED Presentations ( $\mathrm{p}=0.098)$, Inpatient Admissions $(\mathrm{p}=0.936)$, and Inpatient Bed-Days $(\mathrm{p}=$ 0.980) (Table 2). And there was no significant difference between the groups with regard to average age at recruitment $(\mathrm{p}=0.097)$.

\section{Assessment of Patient outcomes}

The Paediatric Asthma Care Givers Quality of Life Questionnaire' (PACQLQ) was completed by the patient's parent/carer if Aged $<7$ yrs, or the PAQLQ completed in consultation with the patient if older. An initial assessment was conducted at recruitment 
$(\mathrm{Ax} 1)$, and repeated at intervals until discharge from the project $(\mathrm{AxF})$. The PACQLQ and PAQLQ are designed to measure the limitations and anxieties faced by caregivers of children with asthma and in the case of the later, the child's perceptions of these issues. They are comprised of 13 items in 2 domains \& categories: (i) Activity limitations (4 items), (ii) Emotional function (9 items). Scoring uses a 7-point Likert scales, with 1 indicating severe impairment and 7 indicating no impairment. The publishers of the questionnaire state that a change of 3.5 is considered to be of clinical importance (Juniper et al., 1996a, 1996b, 1997; Guyatt, 1997). In addition to the quantitative data, carers and some of the older patients were interviewed to ascertain their perceptions of the model of care and its efficacy.

\section{Data analysis}

The general design of the evaluation and analysis of the outcome measures of the project were similar to those used in the coordinated care trials (Smith et al., 2002) and other HARP projects (Bird et al., 2005, 2007, 2010). Hospital demand data were analysed for patients who were offered participation in the project between February 2004 and $1^{\text {st }}$ October 2007 and, who had been recruited to the project for a minimum of 90-days. The criterion of ninety days was selected subjectively, as it was deemed a suitable minimum duration for the interventions of the project to have an observable impact. The same 90day criterion and average length of participation were applied to the 'dummy recruitment' dates of the Comparator Group. The baseline characteristics of the HARP intervention and comparator groups were compared using Kruskal-Wallis tests. Patient outcomes data 
were analysed for patients who had completed a discharge assessment prior to October 2009.

Patient pre-recruitment use of Hospital services, such as the number of Emergency Department presentations, Inpatient Admissions and Bed-days, were determined from Hospital records for the 12 months prior to their recruitment. For the purposes of comparison with post-recruitment data, these data were scaled to rates of service use per day for each patient. Post recruitment rates of Emergency Department presentations, admissions and hospital bed-days were scaled by dividing the number of occurrences by the number of days since the patient had been recruited onto the project. Since data were not normally distributed, pre and post recruitment values were compared using nonparametric Wilcoxon signed rank tests with $\alpha=0.01$, to adjust for multiple comparisons.

For the PAQOL and PACQOL data the group was divided into the age groups $(<5$ years, 5 - 12 years, and >12 years), and their pre-intervention data (Ax1) compared using ANOVA to determine whether any pre-intervention differences were evident. The preand post intervention scores were then compared using a split-plot ANOVA with pre/post comparisons as a within subject factor, and age group as a between subjects factor No PAQOL or PACQOL data were available for the non-HARP comparator group.

The financial implications of the project and potential savings resulting from changes in the use of hospital and community health services were calculated from the budget expenditure reports and the cost of hospital services data derived from Weighted 
Equivalent Inlier Separations (WEIS) reports provided by the hospital performance unit. Estimated savings were calculated for the current size of the HARP cohort and adjusted for the percentage change seen in the non-HARP comparator group.

\section{Results}

Hospital demand data (Tables 2 and 3)

Data were analysed for 223 patients who had agreed to participate in the HARP project. This comprised of 90 female and 133 male patients, with an average age at recruitment of $5.3 \pm 3.5$ years (female $5.4 \pm 3.8$ years and male $5.3 \pm 3.3$ years), and ranged from 1 to 17 years. Pre-recruitment they had an average of 3.0 \pm 1.7 ED Presentations per patient per year, and 184 participants $(82.5 \%)$ had a history of 2 or more ED Presentations in the year prior to recruitment. At the time of this evaluation; 67 patients (30\%) were still actively recruited to the project, 146 had been discharged to GPs and/or other services (65.5\%), 5 withdrawn $(2.2 \%)$ and 5 were no longer contactable. Patients initially declining recruitment when contacted by a Care Facilitator, but consenting to recruitment following a later presentation to ED and contact with a Care Facilitator are included in the HARP group, with their date of recruitment being the date of discharged from the event at which recruitment was successful. Consequently, the HARP group includes some patients with pre- recruitment rates of presentation that are greater than 3 in the previous 12 months. 
Overall, the HARP patients had been recruited to the project for an average of $252.6 \pm$ 122.7 days. The main reason for discharge from the project was the intervention resolving the patients' health care issues, usually through the project facilitating the attainment of the required level of support from community based healthcare services and/or improved self-management.

The Comparator group consisted of 72 patients aged $5.1 \pm 4.3$ years (range 1 - 18 years). It was comprised of 45 male $(4.6 \pm 4.2$ years $)$ and 27 female $(5.9 \pm 4.4$ years $)$ patients. The patients from the Comparator group had an average of 3.6 \pm 2.0 ED presentations per patient per year within the period of 12 months prior to their dummy recruitment. All had a history of 2 or more ED presentations in the year prior to their dummy prerecruitment.

When comparing the pre-recruitment data on use of hospital services (Table 2), no significant differences were identified between the HARP intervention and the Comparator group for: rates of ED Presentations $(\mathrm{p}=0.098)$, Inpatient Admissions $(\mathrm{p}=$ 0.936), and Inpatient Bed-Days $(\mathrm{p}=0.980)$. Additionally, there was no significant difference between the groups with regard to average age at recruitment $(p=0.097)$. Hence, at the time of recruitment/dummy recruitment, the HARP intervention and Comparator groups were not found to differ in their use of hospital services.

The HARP participants $(n=223)$ accounted for 688 ED presentations at the Hospitals within 12 months prior to recruitment and 206 ED presentations post- recruitment. When 
these data were scaled to the patients' length of time on the project, this revealed a statistically significant, $57 \%$ reduction in ED presentation rates per day post recruitment (Wilcoxon's $\mathrm{Z}=-9.7, \mathrm{p}<0.001$ ) (Table 3), with 115 having no ED presentations postrecruitment. This was in contrast with the data for the comparator group, which displayed a $26.5 \%$ increase in ED presentations. Likewise, similar changes and differences were detected in inpatient admissions and hospital bed-days, with the HARP group recording 220 admissions and 342 bed-days in the 12-months pre-recruitment, and declines of $74 \%$ and $71 \%$ respectively, post-recruitment. This was in contrast to the comparator group which, post dummy-recruitment, showed $32 \%$ and $14 \%$ increases respectively. And as a consequence of these diverging rates, the post-intervention rates of ED presentations, Inpatient Admissions and Inpatient Bed-Days of the HARP and Comparator groups were statistically significantly different $(\mathrm{p}<0.001)$ (Table 2$)$.

\section{Patient outcome data (Table 4)}

Data were analysed for four hundred and twenty four participants who had been recruited to the HARP project between October 2004 and September 2008 (155 female and 269 male; aged $4.45 \pm 3.09$ years, range $0.11-17.21$ years), of whom 331 (4.69 \pm 3.19 years, range 0.85 - 17.22 years) had completed a final assessment (AxF). These were completed a mean of $186 \pm 101$ (range 29 -919) days after their first assessment. 
ANOVAs comparing the Ax1 data for the three age groups detected no statistically significant differences in their pre-intervention scores for Activity limitation $(\mathrm{P}>0.9)$ or Emotional Function $(\mathrm{P}>0.6)$. Nor were there statistically significant differences for the length of time between $\mathrm{Ax} 1$ and $\mathrm{AxF}$ ( $<5$ years = 188 days; $5-12$ years =174 days, and $>12$ years $=196$ days; $p=0.704)$.

The split-plot ANOVAs showed a statistically significant and clinically important (>5 points) improvement (pre vs post-intervention) in both Activity Limitation $(\mathrm{p}<0.001)$ and Emotional Function $(\mathrm{p}<0.001)$. And the analyses did not detect any age group by time interaction for either domain ( $p=0.841$ and $p=0.795$ respectively), which indicates that whilst the entire group displayed improvements in both domains, the three age groups were not found to differ in the magnitude of change. However, it is acknowledged by the authors that in the absence of a control group and/or data from the Comparator group these changes cannot be attributed to the model of care and may have occurred naturally over time and/or be related to the timing of patient recruitment into the project, i.e. recently following an ED presentation.

When the cost of the project $(\$ 400 \mathrm{k}-\$ 600 \mathrm{k})$ and additional services were compared alongside the estimated savings (\$597k) attributed to fewer ED presentations, Admissions and Bed-Days, the financial outcome was found to be between cost neutral and an annual saving of $\$ 200 \mathrm{k}$. This broad range spans the worst to best case scenarios, since in this evaluation it was not possible to calculate a more precise figure due to a 
number of the resources and management staff being shared across parallel projects for patients with Chronic Obstructive Pulmonary Disease, Chronic Heart Failure, and Frail Aged (Bird et al., 2007, 2010).

\section{Discussion}

The overall results indicate that carers/patients who participated in this model of care displayed a reduced demand for acute hospital services post-recruitment compared to a similar group who had presented to the hospital in the 3-years prior to the new model of care. Whilst the study design cannot provide irrefutable proof that it was the model of care that caused these improvements there are strong indicators to support a claim that it made a substantive contribution. The reasons for these declines in demands were attributed to carers being better informed of their child's condition, and better prepared to prevent an episode and/or deal with one, as illustrated by the comment by one parent in an interview:

"I feel much more in control of my child's asthma"

These findings concur with those of Sockrider et al. (2006), who reported that following a family tailored self-management intervention, there were significant improvements in carer's self-confidence to deal with an episode, and a reduction in ED visits in the twelve months follow-up. These outcomes are also similar to those described by Khan et al. (2004) who reported increased use of asthma action plans and improved parental asthma knowledge scores to be associated with improved asthma outcomes. 
Based on the interview data, an essential component of the success of the model in this evaluation was the development of the professional and trusting relationship between the Care Facilitator and patient/carer. Carers reported feeling that the Care Facilitator understood their circumstances and likewise through the pre-arranged repeat visits the Care Facilitator developed a strong appreciation of the patient's situation and needs for optimal quality health care. This ensured the most appropriate referral to community health services occurred and enabled the carer(s)/parent(s) to attain the level of understanding of their child's asthma, care plan, and action plan that were needed to optimise their health and a planned, coherent delivery of health services. The importance of the planned repeated contact with the Care facilitator is an interesting issue for many reasons, not least being its potential contribution towards improving carer understanding and subsequent compliance with discharge instructions, in light of the well-established phenomenon of patients with a wide variety of health issues failing to recall much of the discharge instruction provided at ED (Sanderson et al. 2009). It was also likely to facilitate ongoing accessing of the required services, as carers' fostered their own understanding and appreciation of those available to them. Such empowerment is likely to promote a higher quality 'Transition of Care', as identified by Coleman and colleagues (Kripalani et al., 2007; Parrish et al., 2009) and greatly enhancing the overall effectiveness of health service provision. This it should be noted, did not require substantive changes at the point of delivery by GPs or other specialists, but ensured optimisation of their existing services for the benefit of the patient, and through enhanced use of existing relevant health care reduced the need for acute services. 
The magnitude of the reductions in ED presentations, admissions (hospitalizations) and Bed-days (total days in hospital) are similar to that reported in other studies (Cree et al., 2006; Newcomb, 2006; Shelledy et al., 2005) and consequently the model described here provides additional support for such preventative models of care, which can pro-actively reduce hospital demand through an enhanced continuity of care, self-management, and overcoming the barriers to follow-up primary care (Zorc et al., 2005).

Furthermore, whilst it is likely that the study failed to capture all of the patients' use of services pre-recruitment, when for example they may have presented to another hospital, the researchers believe that during the post-recruitment phase, the regular contact between patient and Care Facilitator, which included regular phone monitoring, would result in minimal data loss. Consequently it is possible that any bias in data loss may result in an underestimate of the beneficial impact of the project.

Based on the size of the current group the calculated reductions in hospital demand of the HARP group compared with the recorded increases by the comparator group, estimated annual savings were approximately: 550 ED presentations, 220 hospital admissions, and 300 Bed-days. And despite the aforementioned lack of precision concerning the financial implications of the project, the financial indications appear encouraging, and since this will be an important consideration for healthcare organisations when assessing the potential of new healthcare interventions (Parrish et al., 2009), a more detailed economic evaluation has been proposed. Based upon these and other findings, this project and 
similar ones for other patient groups have subsequently been mainstreamed into the region's healthcare system.

\section{Limitations}

When reporting the results of this project it is appreciated that the findings could be criticized due to the lack of a randomised control group, particularly in the context of changes over time due to maturation. In generating the Comparator Group the authors acknowledge that there are issues relating to the different timeframe of the HARP intervention and non-participant (Comparator) groups, yet the 'real-world' nature of the project prohibited a randomized control design. This was also an issue faced by those evaluating the coordinated care trials (Esterman and Ben-Tovim, 2002) and the Sharing Health Care Initiative Demonstration Projects (Department of Health and Ageing, 2005).

\section{Conclusion}

Compared with the previous delivery of 'all usual care', patients recruited to the HARP project demonstrated substantial reductions in their demand for hospital services and clinically important improvements in their Activity Limitation and Emotional Function scores of the PACQOL and/or PAQLQ. These improvements were attributed to improved carer and/or self-management, linkages to community services, and improved Transition of Care, the combination of which resulted in an overall improved quality of health care. 


\section{References}

Australian Bureau of Statistics: 2001 Census of Population and Housing - SEIFA 2001. http://www.abs.gov.au/websitedbs/D3110124.NSF/f5c7b8fb229cf017ca256973001 fecec/a17dc48d988ecf63ca256dad00005ea3!OpenDocument

Australian Institute of Health and Welfare (2001). Health and community services labour force 2001. National health labour force series No. 27. September 2003; ISSN 1327 4309; ISBN 1740243099

Bird S, Kurowski W and Dickman G (2005). Evaluating a model of service integration for older people with complex health needs. Evaluation Journal of Australasia 4 (1 \& 2), $34-41$.

Bird S R, Kurowski W, Dickman GK and Kronborg I (2007). Integrated care facilitation for older patients with complex health care needs reduces hospital demand. Australian Health Review 31 (3), 451-461

Bird S, Noronha M, Kurowski W, Sinnott H (2010). An integrated care facilitation model improves quality of life and reduces use of hospital resources by patients with chronic obstructive pulmonary disease and chronic heart failure. Australian Journal of Primary Health, 16: 326-333.

Clark NM, Mitchell HE, Rand CS (2009). Effectiveness of Educational and Behavioral Asthma Interventions. Pediatrics, 123:S185-S192.

Cree M, Bell NR, Johnson D and Carriere KC (2006). Increased continuity of care associated with decreased hospital care and emergency department visits for patients with asthma. Disease Management, 9: 63-71. 
Department of Health and Ageing (2005). National Evaluation of the Sharing Health Care Initiative Demonstration Projects, 2005. Canberra: DHA

Department of Human Services (2002). Western Metropolitan Region Health and Social

Wellbeing Profile (2nd ed.), 2002. Melbourne: Department of Human Services.

Department of Human Services (2003). Hospital admission risk program (HARP):

Integrated care for clients with complex needs working party report, 2003.

Melbourne: Hospital Demand Management, Metropolitan Health and Aged Care

Services Division, Victorian Government Department of Human Services.

Department of Human Services (2006). Improving Care: Hospital Admission Risk

Program (Public report): Victorian Government, Melbourne.

Esterman AJ, Ben-Tovim DI (2002). The Australian coordinated care trials: success or failure? Medical Journal of Australia. 177: 469- 470

Guyatt GH, Juniper EF, Griffith LE, Feeny DH, Ferrie PJ. Children and adult perceptions of childhood asthma. Paediatrics 1997;99(2):165-8.

Hopkins S and Speed N. The decline in 'free' general practitioner care in Australia:

reasons and repercussions. Health Policy 2005;73(3):316-29.

Juniper EF, Guyatt GH, Feeny DH, Ferrie PJ, Griffith LE, Townsend M (1996a).

Measuring quality of life in the parents of children with asthma. Quality of Life

Research; 5: 27-34.

Juniper EF, Guyatt GH, Feeny DH, Ferrie PJ, Griffith LE, Townsend M (1996b).

Measuring quality of life in children with asthma. Quality of Life Research; 5:35-46. 
Juniper EF, Guyatt GH, Feeny DH, Griffith LE, Ferrie PJ (1997). Minimum skills required by children to complete health-related quality of life instruments for asthma: comparison of measurement properties. Eur Respir J10(10):2285-94.

Khan MSR, O’Meara M, Stevermuer TL and Henry RL (2004). Randomized controlled trial of asthma education after discharge from an emergency department. Journal of Paediatric Child Health, 40: 674-677.

Kripalani S, Jackson AT, Schnipper JL and Coleman EA. (2007). Promoting effective Transitions of care at Hospital Discharge: A Review of Key Issues for Hospitalists. Journal of Hospital Medicine, 2: 314 - 323.

Newcomb P. (2006). Results of an Asthma Disease Management Program in an Urban Paediatric Community Clinic. Journal for Specialists in Paediatric Nursing, 11: 178 $-188$.

Parrish MM, O’Malley K, Adams RI, Adams SR and Coleman EA. (2009).

Implementation of the Care Transitions Intervention: Sustainability and Lessons Learned. Professional Case Management, 14: 282 - 293.

Reeves MJ, Bohm SR, Korzeniewski SJ and Brown MD. Asthma Care and Management Before an Emergency Department Visit in children in Western Michigan: How well does care adhere to guidelines? Paediatrics, 2006; 117: S118 - S126.

Sanderson BK, Thompson J, Brown TM and Tucker VB (2009). Assessing Patient Recall of Discharge Instructions for Acute Myocardial Infarction. Journal for Healthcare Quality, 31: 25-34. 
Shelledy DC, McCormick SR, LeGrand TS, Cardenas J, and Peters JI (2005). The effect of a paediatric asthma management program provided by respiratory therapists on patient outcomes and cost. Heart and Lung, 34: 423 - 428.

Smith BJ, McElroy HJ, Ruffin RE, Frith PA, Heard AR, Battersby MW, Esterman AJ, Del Fante P, McDonald PJ. (2002). The effectiveness of coordinated care for people with chronic respiratory disease. Medical Journal of Australia, 177(9):4815.

Smith LC, Amsing N, Pilbrow DS, Bird SR, Sinnott HM, Teichtahl H and Orkin CJ (2003). Providing better care for patients with chronic disease. Australian Journal of Primary Care,9(2\&3):119-126.

Sockrider MM, Abranson S, Brooks E, Caviness AC, Pilney S, Koerner C and Macias CG (2006). Delivering Tailored Asthma Family Education in a Paediatric Emergency Department Setting: A Pilot Study. Paediatrics, 117: 135-144.

Spiegel J, Love AS, Wood PR, Griffith M, Taylor KR, Williams SG, Redd SC (2006). The Inner-City Asthma Intervention: description of a community-based implementation of an evidence-based approach to asthma management. Ann Allergy Asthma Immunol, 97: S6-10.

Zorc JJ, Scarfone RJ and Li Y (2005). Predictors of Primary Care Follow-up after a paediatric emergency visit for asthma. Journal of Asthma, 42: 571-576. 
Table 1: Member organizations of the HARP Western Consortium

\begin{tabular}{ll} 
Organisation & Healthcare role \\
\hline Western Health & Acute sector with 3 hospitals \\
ISIS Primary Care & Primary Care \\
Shire of Melton Council & Local Government \\
Djerriwarrh Health Services & Health Service, inc. Primary Care \\
Westgate Division of General Practice & GP Practices \\
Western Melbourne Division of General Practice & GP Practices \\
Westgate Health Co-op & GP Practice and Primary Care \\
Royal District Nursing service & Primary Care Nursing \\
Western Region Health Centre & Primary Care \\
Westbay Alliance PCP & Primary Care Partnership \\
Post Acute Facilitation Unit (PACFU) & Sub-acute services \\
\hline
\end{tabular}


Table 2. Hospital demand data. Standardised Ratios of ED presentations, Inpatient Admissions and Bed-days per patient per day for HARP Intervention group vs Comparator Group, for Pre- and Post- recruitment/dummy recruitment.

\begin{tabular}{|c|c|c|c|c|c|}
\hline & Event & HARP & Comparator & Difference & $P$ \\
\hline \multirow[t]{3}{*}{ Pre-intervention } & ED Presentations & 0.0084 & 0.0098 & $17 \%$ & 0.098 \\
\hline & Admissions & 0.0027 & 0.0028 & $4 \%$ & 0.936 \\
\hline & Bed-days & 0.0042 & 0.0049 & $17 \%$ & 0.980 \\
\hline \multirow[t]{3}{*}{ Post-intervention } & ED Presentations & 0.0036 & 0.0124 & $244 \%$ & $<0.001$ \\
\hline & Admissions & 0.0007 & 0.0037 & $429 \%$ & $<0.001$ \\
\hline & Bed-days & 0.0012 & 0.0056 & $367 \%$ & $<0.001$ \\
\hline
\end{tabular}


Table 3. Hospital demand data. Standardised Ratios of ED presentations, Inpatient Admissions and Bed-days per patient per day Pre- versus Post- recruitment/dummy recruitment: Wilcoxon's Signed Ranks Tests, and pre-intervention rates (HARP v Comparator)

\begin{tabular}{llcccc} 
Group & Event & Pre & Post & Difference & $P$ \\
\hline HARP $(\mathrm{N}=223)$ & ED Presentations & 0.0084 & 0.0036 & $-57.0 \%$ & $<0.001$ \\
& Admissions & 0.0027 & 0.0007 & $-74.0 \%$ & $<0.001$ \\
& Bed-days & 0.0042 & 0.0012 & $-71.4 \%$ & $<0.001$ \\
& & & & & \\
\hline Comparator Group $(\mathrm{N}=72)$ & ED Presentations & 0.0098 & 0.0124 & $+26.5 \%$ & 0.312 \\
& Admissions & 0.0028 & 0.0037 & $+32.1 \%$ & 0.241 \\
& Bed-days & 0.0049 & 0.0056 & $+14.3 \%$ & 0.246 \\
& & & & & \\
\hline
\end{tabular}


Table 4. Changes in PACQOL/PAQOL for the domains of Activity Limitation and Emotional Function for the whole group and subdivided in age groups.

Age Group N Ax1 AxF Mean Difference $\quad \mathrm{P}$

\begin{tabular}{ccccccc}
\hline Activity Limitation & $<5$ yrs & 230 & $20.0 \pm 7.0$ & $25.5 \pm 4.9$ & 5.5 \\
& $5-12$ yrs & 89 & $19.1 \pm 7.2$ & $25.0 \pm 5.2$ & 5.9 & \\
& $>12$ yrs & 12 & $20.8 \pm 8.5$ & $25.6 \pm 3.8$ & 4.8 & \\
& Total & 331 & $19.8 \pm 7.1$ & $25.4 \pm 5.0$ & 5.6 & $<0.001$
\end{tabular}

\begin{tabular}{llllll}
\hline Emotional Function & $<5$ yrs & 230 & $48.9 \pm 11.9$ & $58.0 \pm 9.1$ & 9.1
\end{tabular}

$5-12$ yrs $\quad 89 \quad 47.5 \pm 12.7 \quad 56.9 \pm 9.6 \quad 9.4$

$\begin{array}{lllll}> & \mathrm{yrs} & 12 & 46.3 \pm 14.6 & 53.1 \pm 11.4\end{array}$

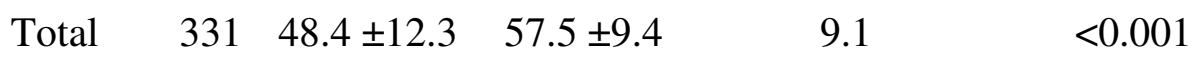

